\title{
BRCA1 missense polymorphisms are associated with poor prognosis of pancreatic cancer patients in a Chinese population
}

\author{
Ying Zhu ${ }^{1, *}$, Kan Zhai ${ }^{2, *}$, Juntao $\mathrm{Ke}^{1}$, Jiaoyuan $\mathrm{Li}^{1}$, Yajie Gong ${ }^{1}$, Yang Yang ${ }^{1}$, Jianbo \\ Tian $^{1}$, Yi Zhang ${ }^{1}$, Danyi Zou ${ }^{1}$, Xiating Peng ${ }^{1}$, Jing Gong ${ }^{1}$, Rong Zhong ${ }^{1}$, Kun Huang ${ }^{3}$, \\ Jiang Chang ${ }^{1}$, Xiaoping Miao ${ }^{1}$ \\ ${ }^{1}$ Key Laboratory of Environment and Health, Ministry of Education and Ministry of Environmental Protection, Tongji Medical \\ College, Huazhong University of Science and Technology, Wuhan, China \\ ${ }^{2}$ Medical Research Center, Beijing Chao-Yang Hospital, Capital Medical University, Beijing, China \\ ${ }^{3}$ Tongji School of Pharmacy, Huazhong University of Science and Technology, Wuhan, China \\ *These authors contribute equally to this work \\ Correspondence to: Jiang Chang, email: changjiang815@hust.edu.cn \\ Xiaoping Miao, email: Miaoxp@hust.edu.cn
}

Keywords: prognosis, pancreatic cancer, BRCA, SNP

Received: February 09, 2017 Accepted: March 15, 2017 Published: March 21, 2017

Copyright: Zhu et al. This is an open-access article distributed under the terms of the Creative Commons Attribution License (CC-BY), which permits unrestricted use, distribution, and reproduction in any medium, provided the original author and source are credited.

\section{ABSTRACT}

Pancreatic cancer is a highly lethal disease with limited prognostic marker. BRAC1 and BRCA2 are two classic tumor suppressor genes which play an important role in DNA repair. Somatic mutations and germline genetic variants on $B R C A 1 / 2$ have been found associated with the tumorigenesis of pancreatic cancer. However, the correlations between BRCA1/2 polymorphism and pancreatic cancer prognosis remained unknown. In this study, we genotyped three tag missense variants on $B R C A 1 / 2$ in 603 sporadic pancreatic cancer patients in a Chinese population. We found rs1799966 on BRCA1 was associated with poor prognosis of pancreatic cancer patients with hazard ratio being $1.23(95 \% \mathrm{CI}$ : 1.09-1.40, $P=0.0010)$. Further stratification analyses showed that significant correlation was particularly in locally advanced stage patients with hazard ratio being 1.36 (95\% CI: 1.13-1.64, $P=0.0014)$, but not in patients in local stage $(P=0.1139)$ or metastatic stage $(P=0.5185)$. Two missense variants (rs766173 and rs144848) on BRAC2 showed no significant correlation with pancreatic cancer patients' overall survival. In conclusion, we identified a germline missense variant on BRAC1 significantly associated with poor prognosis of pancreatic cancer patients with locally advanced stage. These results may contribute to the precision medicine of this disease.

\section{INTRODUCTION}

Pancreatic cancer is the thirteenth most common cancer in the world with mortality closely parallels incidence $[1,2]$. Despite decades of efforts, there are still few early detection methods and effective treatments of this disease. Nevertheless, patient survival times are varied and only partly explained by traditional clinical and pathological features [3]. Accumulating evidences have shown that germline variation such as single nucleotide polymorphisms (SNP) can not only affect cancer susceptibility [4, 5], but also confer patients with different prognosis in multiple cancers [6-8]. Genetic variation relating to transcription regulation and DNA damage repair have effects on patient survival particularly.

$B R C A 1$ and BRCA2 are two recognized tumor suppressor genes, for the encoded proteins play important roles in transcription and DNA repair of double-stranded breaks via homologous recombination $[9,10]$. Mutations in $B R C A 1 / 2$ have been confirmed to give rise to multiple cancers including pancreatic adenocarcinoma [11-14]. Moreover, prognostic value of germline $B R C A$ mutations have also been found in breast, ovarian, prostate and other cancers [15-19]. Germline variants on BRCA2 have also been identified to be associated with pancreatic cancer risk [20]. However, correlation between variants on $B R C A$ and pancreatic cancer patients' survival are rarely studied. 
In the present study, we investigated the impact of germline missense variants on $B R C A 1 / 2$ and overall survival (OS) of pancreatic cancer patients. Three tag missense polymorphisms (rs1799966 on BRCA1; rs 766173 and rs 144848 on $B R C A 2$ ) were genotyped in 603 pancreatic cancer patients in a Chinese population.

\section{RESULTS}

\section{Characteristics of study subjects}

The characteristics of the 603 pancreatic cancer patients are shown in Table 1. Median follow-up time for these patients was 15 months. 523 (86.7\%) patients died during our follow-up time with median survival time (MST) being 7.4 months. As expected, disease stage was strongly associated with patients' overall survival $(P<0.0001)$ with MST being 13.7, 8.1 and 4.4 months for patients with local, locally advanced and metastatic stage, respectively. Patients' gender, age, smoking or drinking status showed no significant correlation with patients' overall survival.

\section{Genetic variants on $B R C A 1$ is associated with pancreatic patients' overall survival}

We retrieved a total of 24 missense variants with global MAF greater than 0.01 on BRCA1 and BRCA2 (Supplementary Table 1). Among these variants, seven (four on BRCA1 and three on BRCA2) are common variants with $\mathrm{MAF}>0.05$ in $\mathrm{CHB}$ (Chinese Han, Beijing) population (Supplementary Table 1). All the four missense variants on BRCA1 (rs1799966, rs16942, rs16941, rs799917) are in perfect linkage disequilibrium (LD) (Figure 1). For BRCA2, rs766173 and rs1799944 are in complete LD, but independent with the rest variant rs144848 (Figure 1). Therefore, considering the LD relations and function prediction clues provided by SIFT and Polyphen, we selected rs 1799966 (BRCA1), rs766173 and rs144848 (BRCA2) as tag SNPs for genotying and further survival analysis. As a result, we observed that rs1799966 (BRCA1) was significantly associated with pancreatic cancer patients' overall survival with HR being 1.24 under an additive model (95\% CI: 1.09-1.40,

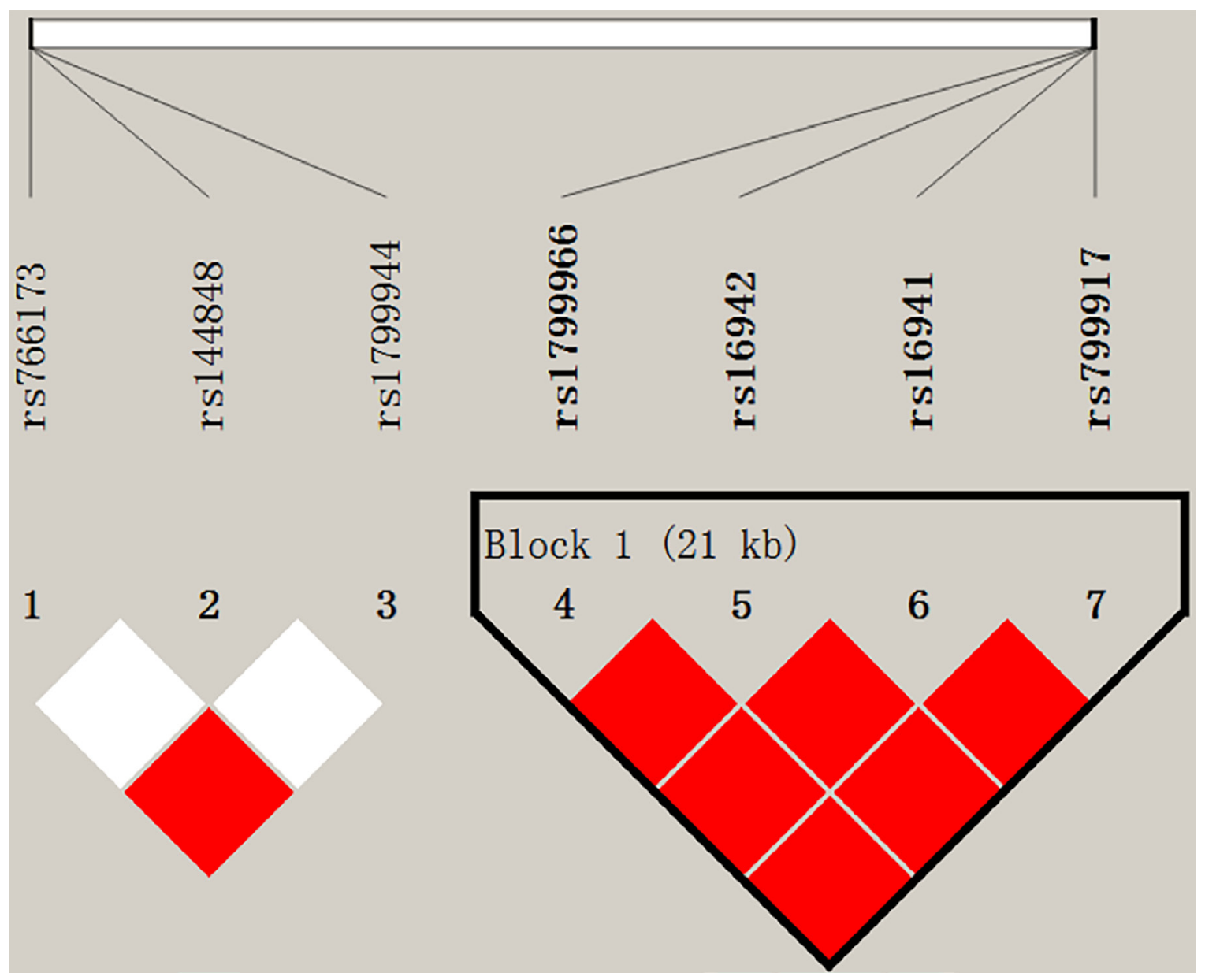

Figure 1: Linkage disequilibrium (LD) of SNPs at the BRCA1/2 gene locus. rs 766173 and rs 1799944 on $B R C A 2$ are in complete linkage disequilibrium (LD), but independent with the rest SNP rs144848. Four SNPs (rs1799966, rs16942, rs16941 and rs799917) on $B R C A 1$ are in complete LD. The LD plot was based on 1000 genome phase 3 data among the Han Chinese in Beijing (CHB). White diamond indicates hardly no evidence of $\operatorname{LD}\left(r^{2}=0.028\right)$, while red indicates strong evidence $\left(r^{2}=1\right)$. 


\begin{tabular}{lccc}
\hline & No. $(\%)$ & MST & $\boldsymbol{P}^{\dagger}$ \\
\hline Vital status & & & \\
Dead & $523(86.7)$ & & \\
Alive & $80(13.3)$ & & \\
Gender & & 7.6639 \\
Male & $369(61.2)$ & 7.4 & \\
Female & $234(38.8)$ & & \\
Age & & 7.0848 \\
$\leq 61$ & $309(51.2)$ & 7.1 & \\
$>61$ & $294(48.8)$ & & \\
Smoking status & & 7.0 & \\
Yes & $140(23.2)$ & 7.5 & \\
No & $463(76.8)$ & & \\
Drinking status & & 7.6 & \\
Yes & $108(17.9)$ & 7.3 & \\
No & $495(82.1)$ & & \\
Clinical stage & & 13.7 & \\
Local & $137(22.7)$ & 8.1 & \\
Locally advanced & $268(44.5)$ & 4.4 & \\
Metastatic & $198(32.8)$ & & \\
\hline
\end{tabular}

Note: MST, median survival time (months).

$\dagger P$ values were calculated using the log-rank test.

$\$$ Median age was 61 years among all cases.

$P=0.0010$ ) (Table 2 and Figure 2). Comparing with patients with AA genotype, the patients with AG genotype and GG genotype were significantly associated with poor prognosis with median survival time (MST) being 7.5 month $(\mathrm{HR}=1.32,95 \% \mathrm{CI}: 1.09-1.59$, $P=0.0044)$ and 6.7 month (HR $=1.41,95 \%$ CI: $1.07-1.85$, $P=0.0143$ ), respectively (Table 2). However, two variants on BRCA2 (rs766173 and rs144848) showed no significant correlation with prognosis of pancreatic cancer patients with $P$ values under an additive model being 0.8579 and 0.1798 , respectively (Table 2 and Figure 2 ).

\section{Stratification analyses of rs1799966 with pancreatic cancer patients' overall survival}

We further performed stratified analyses by gender age, smoking status, drinking status and clinical stage to evaluate the effects of rs1799966 (c.4837A $>\mathrm{G}$ [p.Ser1613Gly]) on pancreatic cancer patients' overall survival (Table 3 and Supplementary Table 2). The rs1799966 showed significant association with overall survival of pancreatic cancer patients with different age group ( $P=0.0095$ and $P=0.0370$ for patients $\leq 61$ years and $>61$ years, respectively), smoking status $(P=0.0025$ and $P=0.0210$ for smoker and non-smoker, respectively) and drinking status $(P=0.0144$ and $P=0.0117$ for drinker and non-drinker, respectively). The association was also significant in males $(P=0.0014)$ but not in females $(P=0.1654)$. Among patients with different clinical stage, the rs 1799966 was particularly significant associated with poor prognosis of locally advanced stage patients with an HR being 1.36 under an additive model (95\% CI: $1.13-$ 1.64, $P=0.0014$ ) (Table 3 and Figure 3 ) and no significant correlation between rs1799966 and patients' overall survival was showed in patients with local $(P=0.1139)$ or metastatic stage $(P=0.5185)$ (Table 3 and Figure 3$)$.

\section{DISCUSSION}

In this study, we interrogate the correlation between germline missense variants on $B R C A 1 / 2$ and pancreatic cancer patients' overall survival. Through genotyping of three tag missense variants on BRCA1/2 in 603 Chinese pancreatic cancer patients, we discovered the prognostic value of rs1799966 (c.4837A $>$ G [p.Ser1613Gly]) on BRCA1. Further stratification analyses showed that the association was particularly in patients with local advanced stage, but not patients in local or metastatic stage. 
Table 2: Association between variants on BRCA1/2 and PDAC prognosis

\begin{tabular}{|c|c|c|c|c|}
\hline SNP, Gene & No. $(\%)$ & MST & HR (95\% CI) & $\boldsymbol{P}^{\dagger}$ \\
\hline \multicolumn{5}{|l|}{ rs1799966, BRCA1 } \\
\hline $\mathbf{A A}$ & $248(41.1)$ & 7.6 & 1.00 (Reference) & \\
\hline AG & $275(45.6)$ & 7.5 & $1.32(1.09-1.59)$ & 0.0044 \\
\hline GG & $80(13.3)$ & 6.7 & $1.41(1.07-1.85)$ & 0.0143 \\
\hline Additive model & & & $1.23(1.09-1.40)$ & 0.0010 \\
\hline \multicolumn{5}{|l|}{ rs766173, $B R C A 2$} \\
\hline AA & $487(80.6)$ & 7.4 & 1.00 (Reference) & \\
\hline $\mathrm{AC}$ & $112(18.6)$ & 7.3 & $1.01(0.81-1.26)$ & 0.9302 \\
\hline $\mathrm{CC}$ & $4(0.7)$ & 12.0 & $1.15(0.43-3.10)$ & 0.7767 \\
\hline Additive model & & & $1.02(0.83-1.25)$ & 0.8579 \\
\hline \multicolumn{5}{|l|}{ rs144848, $B R C A 2$} \\
\hline AA & $327(54.2)$ & 7.7 & 1.00 (Reference) & \\
\hline $\mathrm{AC}$ & $235(39.0)$ & 7.1 & $1.08(0.90-1.30)$ & 0.3924 \\
\hline $\mathrm{CC}$ & $41(6.8)$ & 6.8 & $1.23(0.86-1.76)$ & 0.2644 \\
\hline Additive model & & & $1.10(0.96-1.27)$ & 0.1798 \\
\hline
\end{tabular}

Note: SNP, single nucleotide polymorphism; MST, median survival time (months); HR, hazard ratio; CI: confidence interval. $\dagger P$ values were calculated using Cox regression adjusting for gender, age, smoking, drinking status and clinical stage.

$B R C A 1$ and $B R C A 2$ are two classic tumor suppressor genes that play important roles not only in tumorigenesis but also cancer progression and outcome. A meta-analysis among 297,402 patients revealed that $B R C A$ mutations were associated with worse overall survival in breast cancer patients [21]. Similar findings were also reported in prostate cancer that significant longer cause-specific overall survival has been reported for $B R C A$ mutation non-carriers compared with carriers [22]. However, in the context of pancreatic cancer, there still lacks convincing epidemiology data or association study for the prognostic effects of $B R C A$ germline mutation. In this present study, we found a tag missense variant (c.4837A $>\mathrm{G}$
[p.Ser1613Gly]) on BRCA1 significantly associated with poor prognosis of pancreatic cancer patients in a Chinese population. This variant can lead to amino acid change from serine to glycine (p.Ser1613Gly), and was predicted to have a possibly damaging of BRCA1 protein function by SIFT or Polyphen. The BRCA1 has many important functions in maintaining the normal physiology of cell and organisms $[23,24]$. Its major role is involved in the regulation of cell cycle checkpoints in response to DNA damage, including the repair of DNA double-strand breaks (DSB) via homologous recombination $[10,25] . B R C A 1$ deficient cells lacking HR activity could accumulate DNA DSB, resulting in genomic instability, malignant
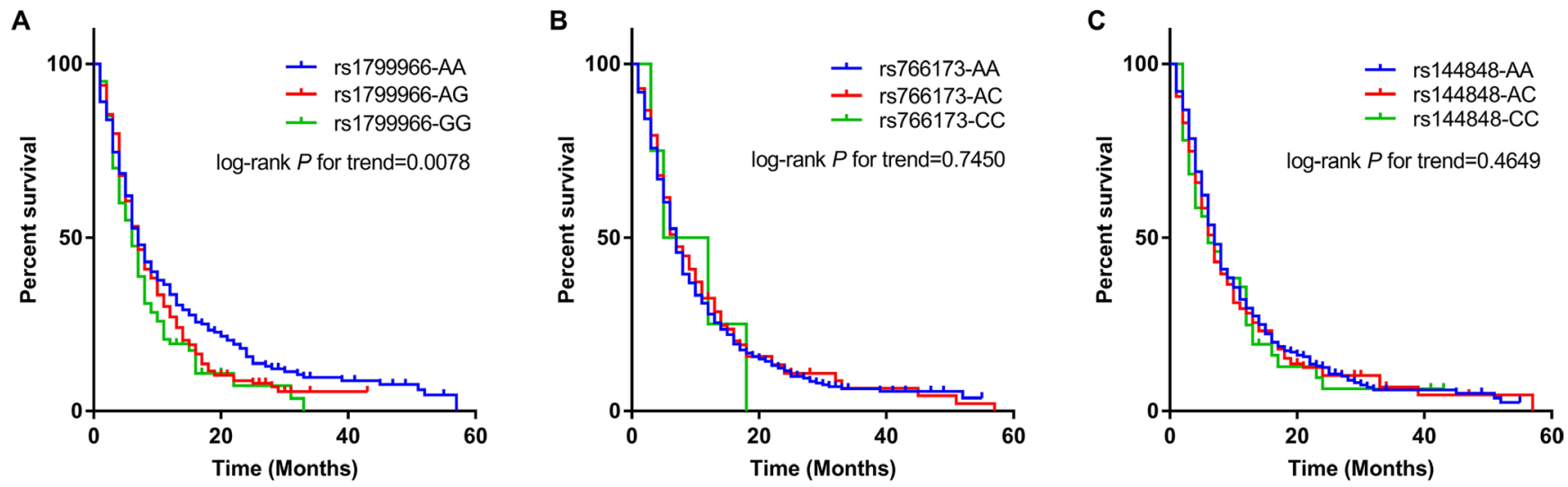

Figure 2: Kaplan-Meier curves of overall survival by rs1799966 (A), rs766173 (B) and rs144848 (C) genotypes. $P$ values were calculated using Log-rank test for trend in an additive model. 
Table 3: Stratification analyses of rs1799966 with PDAC patients' prognosis in different stages

\begin{tabular}{|c|c|c|c|c|}
\hline & No. $(\%)$ & MST & HR $(95 \% \text { CI })^{\dagger}$ & $\boldsymbol{P}^{\dagger}$ \\
\hline \multicolumn{5}{|c|}{ Local disease $(N=137)$} \\
\hline $\mathrm{AA}$ & $49(35.8)$ & 15.0 & 1.00 (Reference) & \\
\hline $\mathrm{AG}$ & $69(50.3)$ & 13.7 & $1.07(0.67-1.69)$ & 0.7858 \\
\hline GG & $19(13.9)$ & 8.8 & $1.70(0.93-3.13)$ & 0.0869 \\
\hline Additive model & & & $1.28(0.94-1.74)$ & 0.1139 \\
\hline \multicolumn{5}{|c|}{ Locally advanced disease $(N=268)$} \\
\hline $\mathbf{A A}$ & $113(42.2)$ & 9.0 & 1.00 (Reference) & \\
\hline AG & $118(44.0)$ & 8.1 & $1.45(1.09-1.94)$ & 0.0104 \\
\hline GG & 37 (13.8) & 6.6 & $1.66(1.11-2.50)$ & 0.0147 \\
\hline Additive model & & & $1.36(1.13-1.64)$ & 0.0014 \\
\hline \multicolumn{5}{|c|}{ Metastatic disease $(N=198)$} \\
\hline AA & $86(43.4)$ & 4.6 & 1.00 (Reference) & \\
\hline $\mathrm{AG}$ & $88(44.5)$ & 4.4 & $1.25(1.92-1.71)$ & 0.1534 \\
\hline GG & $24(12.1)$ & 4.0 & $0.99(0.61-1.61)$ & 0.9703 \\
\hline Additive model & & & $1.07(0.87-1.32)$ & 0.5185 \\
\hline
\end{tabular}

Note: MST, median survival time (months); HR, hazard ratio; CI: confidence interval.

$\dagger P$ values were calculated using Cox regression adjusting for gender, age, smoking and drinking status.

transformation and progression [26]. Accordingly, we conjectured that the missense variants rs1799966 may hinder the performance of $B R C A 1$ in the above biological process thus conferring pancreatic cancer patients in different genotypes with different risk of deterioration and survival time.

In the stratification analyses, we only observed that rs1799966 was significantly associated with survival of locally advanced stage patients. For local stage disease, patients carrying risk allele and genotype also had poorer survival (AG, $\mathrm{HR}=1.07$; GG, $\mathrm{HR}=1.70$ ). However, associations were not significant might because relatively small sample size, and other treatments such as chemotherapy and radiotherapy after surgery which might influence survival times. As for distant metastatic disease, the prognosis are extremely poor for both risk allele carriers and non-carriers, that the genetic effects on patients' survival might not be obvious.

To the best of our knowledge, it was the first association study of $B R C A$ germline variants and pancreatic cancer patients' overall survival. Our work highlighted the important prognostic and predictive value of BRCA1 germline variants, which had been mostly regarded as a risk biomarker of pancreatic cancer though. There were still some limitations for this study. First, our findings need to be validate in replication cohorts with
A

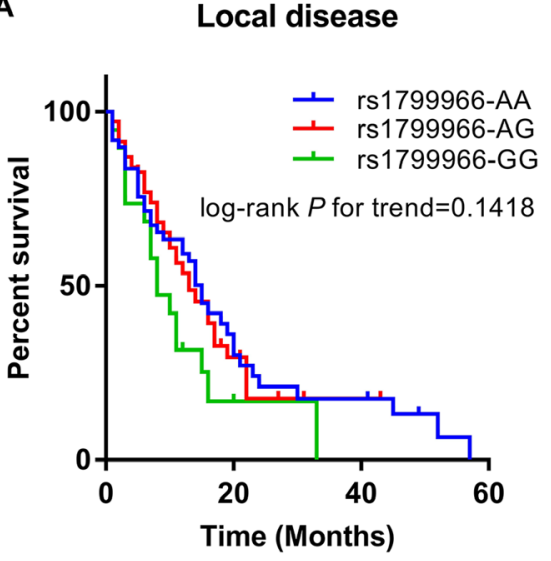

B

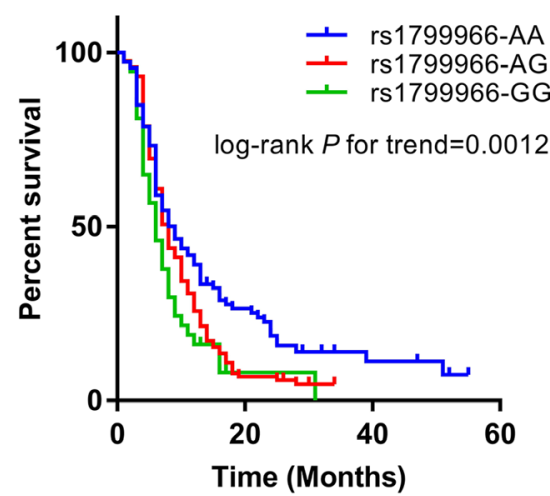

C Metastatic disease

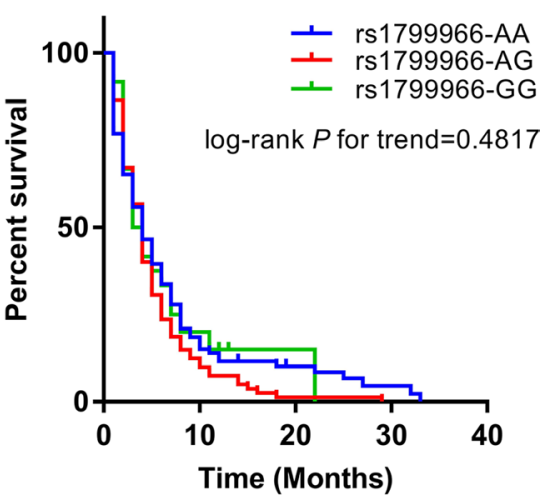

Figure 3: Kaplan-Meier curves by rs1799966 genotypes in different disease stages. $P$ values were calculated using Log-rank test for trend in an additive model in patients with local stage (A), locally advanced stage (B) and metastatic stage (C), respectively. 
larger sample sizes or other populations in the future. Second, the underlying biological mechanism between rs1799966 and pancreatic cancer patients' survival needs further investigation.

In conclusion, we identified a missense variant rs1799966 on BRCA1 associated with a worse overall survival of pancreatic cancer patients, especially in patients with locally advanced stage. Our findings not only lead to new insights into the progression of pancreatic cancer, but also suggest a target for therapy and potential biomarker for prognosis prediction.

\section{MATERIALS AND METHODS}

\section{Study subjects}

Pancreatic cancer patients were recruited from Tongji Hospital of Huazhong University of Science and Technology, Wuhan, China. A subset of individuals were included in our previous studies $[27,28]$. The diagnosis of pancreatic cancer was confirmed by two local pathologists according to the World Health Organization classification [29]. Demographic, clinical and survival information were obtained from the medical records and telephone interview. Informed consent was obtained from all participants at recruitment, and this study was approved by the ethics committee of Tongji Medical College, Huazhong University of Science and Technology.

\section{SNP selection}

We utilized Ensembl genome browser (http:/asia. ensembl.org/index.html) to retrieve all the missense variants on BRCA1 and BRCA2, with global minor allele frequency (MAF) greater than 0.01 (using 1000 genome phase 3 data). Among which, we focused on the common variants in CHB (Han Chinese in Beijing, China) population with MAF in CHB greater than 0.05 (a total of seven variants met the standard, four on $B R C A 1$ and three on BRCA2. Supplementary Table 1.). Then we took advantage of SIFT and PolyPhen to predict variant effects on protein function, and meanwhile measured linkage disequilibrium (LD) relations between each pair of variants using 1000 genome phase 3 data (Figure 1). Synthesizing the above results, we selected 3 tagSNPs (rs1799966 on BRCA1; rs766173 and rs144848 on $B R C A 2$ ) with most likely functional as candidate for genotyping.

\section{Genotyping}

At recruitment, $2 \mathrm{~mL}$ peripheral venous blood sample was collected from each subject with informed consent. Genomic DNA was extracted using the RelaxGene Blood DNA System DP319-02 (TIANGEN). The above three candidate SNPs were subsequently genotyped using a TaqMan assay on the ABI PRISM
7900 HT platform (Applied Biosystems, Inc.). The sequences of primers and TaqMan probes for genotyping candidate SNPs are listed in Supplementary Table 3. For quality control, $5 \%$ duplicate samples were independently reanalyzed in a blinded fashion. The call rate of each SNP was over $95 \%$.

\section{Statistical analysis}

The overall survival time was defined as the time from pancreatic cancer diagnosis to either death or the last known date alive. Cox proportional hazards regression under a log-additive genetic model with adjustment for gender, age, smoking and drinking status and stage of disease was used to measure the effects of candidate SNPs on survival. Haploview software was used to determine pair-wise LD relations. Kaplan-Meier survival estimates were plotted and $P$ values were assessed using the logrank test for trend. Survival analyses were performed with R (3.3.0) using "coxph" function in "Survival package". For all analyses, statistical significance was set at $P<0.05$ and all tests were two sided.

\section{CONFLICTS OF INTEREST}

The authors declare no conflicts of interest.

\section{GRANT SUPPORT}

This work is supported by National Natural Science Foundation of China NSFC-81673256, National Program for Support of Top-notch Young Professionals, National High-Tech Research and Development Program of China 2014AA020609, and Specialized Research Fund for the Doctoral Program of Higher Education 20130142110017 for Xiaoping Miao.

\section{REFERENCES}

1. Siegel RL, Miller KD, Jemal A. Cancer Statistics, 2017. CA Cancer J Clin. 2017; 67:7-30.

2. Chen W, Zheng R, Baade PD, Zhang S, Zeng H, Bray F, Jemal A, Yu XQ, He J. Cancer statistics in China, 2015. CA Cancer J Clin. 2016; 66:115-132.

3. Hidalgo M. Pancreatic cancer. N Engl J Med. 2010; 362:1605-1617.

4. Li J, Zou L, Zhou Y, Li L, Zhu Y, Yang Y, Gong Y, Lou J, Ke J, Zhang Y, Tian J, Zou D, Peng X, et al. A lowfrequency variant in SMAD7 modulates TGF-beta signaling and confers risk for colorectal cancer in Chinese population. Mol Carcinog. 2017.

5. Lou J, Gong J, Ke J, Tian J, Zhang Y, Li J, Yang Y, Zhu Y, Gong Y, Li L, Chang J, Zhong R, Miao X. A functional polymorphism located at transcription factor binding sites, rs6695837 near LAMC1 gene, confers risk of colorectal 
cancer in Chinese populations. Carcinogenesis. 2016; 38:177-183.

6. Wu C, Li D, Jia W, Hu Z, Zhou Y, Yu D, Tong T, Wang M, Lin D, Qiao Y, Zhou Y, Chang J, Zhai K, et al. Genome-wide association study identifies common variants in SLC39A6 associated with length of survival in esophageal squamouscell carcinoma. Nat Genet. 2013; 45:632-638.

7. Dong QZ, Zhang XF, Zhao Y, Jia HL, Zhou HJ, Dai C, Sun HJ, Qin Y, Zhang WD, Ren N, Ye QH, Qin LX. Osteopontin promoter polymorphisms at locus -443 significantly affect the metastasis and prognosis of human hepatocellular carcinoma. Hepatology. 2013; 57:1024-1034.

8. Gong J, Tian J, Lou J, Ke J, Li L, Li J, Yang Y, Gong Y, Zhu Y, Zhang Y, Zhong R, Chang J, Miao X. A functional polymorphism in lnc-LAMC2-1:1 confers risk of colorectal cancer by affecting miRNA binding. Carcinogenesis. 2016; 37:443-451.

9. Prat J, Ribe A, Gallardo A. Hereditary ovarian cancer. Hum pathol. 2005; 36:861-870.

10. Venkitaraman AR. Cancer susceptibility and the functions of BRCA1 and BRCA2. Cell. 2002; 108:171-182.

11. Narod SA. BRCA mutations in the management of breast cancer: the state of the art. Nat Rev Clin Oncol. 2010; 7:702-707.

12. Iqbal J, Ragone A, Lubinski J, Lynch HT, Moller P, Ghadirian P, Foulkes WD, Armel S, Eisen A, Neuhausen SL, Senter L, Singer CF, Ainsworth P, et al. The incidence of pancreatic cancer in BRCA1 and BRCA2 mutation carriers. Br J Cancer. 2012; 107:2005-2009.

13. Greer JB, Whitcomb DC. Role of BRCA1 and BRCA2 mutations in pancreatic cancer. Gut. 2007; 56:601-605.

14. Mersch J, Jackson MA, Park M, Nebgen D, Peterson SK, Singletary C, Arun BK, Litton JK. Cancers associated with BRCA1 and BRCA2 mutations other than breast and ovarian. Cancer. 2015; 121:269-275.

15. Chetrit A, Hirsh-Yechezkel G, Ben-David Y, Lubin F, Friedman E, Sadetzki S. Effect of BRCA1/2 mutations on long-term survival of patients with invasive ovarian cancer: the national Israeli study of ovarian cancer. J Clin Oncol. 2008; 26:20-25.

16. Robson ME, Chappuis PO, Satagopan J, Wong N, Boyd J, Goffin JR, Hudis C, Roberge D, Norton L, Begin LR, Offit K, Foulkes WD. A combined analysis of outcome following breast cancer: differences in survival based on BRCA1/ BRCA2 mutation status and administration of adjuvant treatment. Breast Cancer Res. 2004; 6:R8-R17.

17. Lee EH, Park SK, Park B, Kim SW, Lee MH, Ahn SH, Son BH, Yoo KY, Kang D, Group KR, Korean Breast Cancer S. Effect of BRCA1/2 mutation on short-term and long-term breast cancer survival: a systematic review and meta-analysis. Breast Cancer Res Treat. 2010; 122:11-25.

18. Chappuis PO, Kapusta L, Begin LR, Wong N, Brunet JS, Narod SA, Slingerland J, Foulkes WD. Germline BRCA1/2 mutations and p27(Kip1) protein levels independently predict outcome after breast cancer. J Clin Oncol. 2000; 18:4045-4052.

19. Castro E, Goh C, Leongamornlert D, Saunders E, Tymrakiewicz M, Dadaev T, Govindasami K, Guy M, Ellis S, Frost D, Bancroft E, Cole T, Tischkowitz M, et al. Effect of BRCA Mutations on Metastatic Relapse and Causespecific Survival After Radical Treatment for Localised Prostate Cancer. Eur Urol. 2015; 68:186-193.

20. Huang L, Wu C, Yu D, Wang C, Che X, Miao X, Zhai K, Chang J, Jiang G, Yang X, Cao G, Hu Z, Zhou Y, et al. Identification of common variants in BRCA2 and MAP2K4 for susceptibility to sporadic pancreatic cancer. Carcinogenesis. 2013; 34:1001-1005.

21. Zhu Y, Wu J, Zhang C, Sun S, Zhang J, Liu W, Huang J, Zhang Z. BRCA mutations and survival in breast cancer: an updated systematic review and meta-analysis. Oncotarget. 2016; 7:70113-70127. doi: 10.18632/oncotarget.12158.

22. Castro E, Goh C, Olmos D, Saunders E, Leongamornlert D, Tymrakiewicz M, Mahmud N, Dadaev T, Govindasami K, Guy M, Sawyer E, Wilkinson R, Ardern-Jones A, et al. Germline BRCA mutations are associated with higher risk of nodal involvement, distant metastasis, and poor survival outcomes in prostate cancer. J Clin Oncol. 2013; 31:1748-1757.

23. Quinn JE, Kennedy RD, Mullan PB, Gilmore PM, Carty M, Johnston PG, Harkin DP. BRCA1 functions as a differential modulator of chemotherapy-induced apoptosis. Cancer Res. 2003; 63:6221-6228.

24. Lafarge S, Sylvain V, Ferrara M, Bignon YJ. Inhibition of BRCA1 leads to increased chemoresistance to microtubuleinterfering agents, an effect that involves the JNK pathway. Oncogene. 2001; 20:6597-6606.

25. Moynahan ME, Jasin M. Mitotic homologous recombination maintains genomic stability and suppresses tumorigenesis. Nat Rev Mol Cell Biol. 2010; 11:196-207.

26. Tutt A, Ashworth A. The relationship between the roles of BRCA genes in DNA repair and cancer predisposition. Trends Mol Med. 2002; 8:571-576.

27. Zhu B, Tian J, Zhong R, Tian Y, Chen W, Qian J, Zou L, Xiao M, Shen N, Yang H, Lou J, Qiu Q, Ke J, et al. Genetic Variants in the SWI/SNF Complex and Smoking Collaborate to Modify the Risk of Pancreatic Cancer in a Chinese Population. Mol Carcinogen. 2014; 54:761-768.

28. Zhu B, Zhu Y, Lou J, Ke J, Zhang Y, Li J, Gong Y, Yang Y, Tian J, Peng X, Zou D, Zhong R, Gong J, et al. A single nucleotide polymorphism in the 3'-UTR of STAT3 regulates its expression and reduces risk of pancreatic cancer in a Chinese population. Oncotarget. 2016. Oncotarget. 2016; 7:62305-62311. doi: 10.18632/oncotarget.11607.

29. Hruban RH BeP, Hiraoka N, et al. Ductal adenocarcinoma of the pancreas. In: WHO classification of tumours of the digestive system. 2010; pp. 281-291. 